\title{
Variational Continuous Assimilation of TMI and SSM/I Rain Rates: Impact on GEOS-3 Hurricane Analyses and Forecasts
}

\author{
Arthur Y. Hou*, Sara Q. Zhang', Oreste Reale, \\ National Aeronautics and Space Administration/Goddard Space Flight Center, Greenbelt, Maryland. \\ +Science Applications Intemational Corp., Beltsville, Maryland. \\ "University of Maryland Baltimore County, Baltimore, Maryland.
}

Summary

We have developed a variational continuous assimilation (VCA) algorithm for precipitation assimilation and examined its effectiveness in using 6-h rain rates from TRMM Microwave Imager (TMM) and Special Sensor Microwave/Imager (SSM//) to improve global analyses and forecasts of tropical hurricanes. In contrast to other variational techniques, this scheme uses moisture and temperature tendency corrections as the control variable to offset model deficiencies. For rainfall assimilation, forecast model errors are of special concern since model-predicted precipitation is based on parameterized moist physics, which can have substantial systematic errors.

We performed parallel assimilation experiments with and without rainfall data in the Goddard Earth Observing System Version 3 (GEOS-3) global data assimilation system (DAS) to examine the impact precipitation data on analyses and forecasts of synoptic features of prominent tropical weather systems using Hurricanes Bonnie and Floyd as case studies. Results show that assimilating 6-h TMI and SSM/1 surface rain rates leads to more realistic storm structures through improved rainfall analysis. Forecast experiments show that the improved analysis provide better initial conditions for 5 -day track prediction and precipitation forecast. The improved forecast skills were confirmed in a sensitivity study that showed a systematic increase in forecast skills as more rainfall information was retained in the initial condition.

Results of this study suggest that addressing model deficiencies in moisture tendency may be crucial to making effective use of precipitation observations in data assimilation. They also show that the full benefit of precipitation data can be realized during an assimilation cycle only if analyses of wind, temperature, moisture, and pressure are allowed to respond to an improved rainfall analysis. More generally, this VCA tendency correction procedure may be incorporated as a part of an augmented control variable within the framework of a four-dimensional variational analysis or as a model error estimator in sequential analysis schemes. 


\title{
Variational Continuous Assimilation of TMI and SSM/I Rain Rates: Impact on GEOS-3 Hurricane Analyses and Forecasts
}

\author{
Arthur Y. Hou, Sara Q. Zhang*, Oreste Reale ${ }^{+}$ \\ Global Modeling and Assimilation Office \\ NASA Goddard Space Flight Center, Greenbelt, MD 20771
}

June 16, 2003

(Manuscript for Monthly Weather Review)

* Additional affiliation: Science Applications International Corp., Beltsville, Maryland.

${ }^{+}$Additional affiliation: University of Maryland Baltimore County, Baltimore, Maryland.

Corresponding author address: Dr. Arthur Y. Hou, Code 900.3, NASA Goddard Space Flight Center, Greenbelt, MD, 20771.E-mail: arthur.y.hou@nasa.gov 


\begin{abstract}
We describe a variational continuous assimilation (VCA) algorithm for assimilating tropical rainfall data using moisture and temperature tendency corrections as the control variable to offset model deficiencies. For rainfall assimilation, model errors are of special concern since model-predicted precipitation is based on parameterized moist physics, which can have substantial systematic errors. This study examines whether a VCA scheme using the forecast model as a weak constraint offers an effective pathway to precipitation assimilation.

The particular scheme we examine employs a " $1+1$ " dimension precipitation observation operator based on a 6-h integration of a column model of moist physics from the Goddard Earth Observing System (GEOS) global data assimilation system (DAS). In earlier studies, we tested a simplified version of this scheme and obtained improved monthly-mean analyses and better short-range forecast skills. This paper describes the full implementation of the 1+1D VCA scheme using background and observation error statistics, and examines how it may improve GEOS analyses and forecasts of prominent tropical weather systems such as hurricanes.

Parallel assimilation experiments with and without rainfall data for Hurricanes Bonnie and Floyd show that assimilating 6-h TMI and SSM/I surface rain rates leads to more realistic storm features in the analysis, which, in turn, provide better initial conditions for 5-day storm track prediction and precipitation forecast. These results provide evidence that addressing model deficiencies in moisture tendency may be crucial to making effective use of precipitation information in data assimilation.
\end{abstract}




\section{Introduction}

Precipitation estimates from space-borne passive microwave instruments such as the TRMM Microwave Imager (TMI) and Special Sensor-Microwave/Imager (SSM/I) have been assimilated using a variety of techniques to improve global atmospheric analyses and forecasts (e.g., Krishnamurti et al. 1993, Tsuyuki 1997, Treadon 1997, Hou et al. 2000, Marecal and Mahfouf2002). Currently, operational global weather forecast systems typically use a multi-dimensional variational scheme to optimize the initial condition of a forecast. These schemes assume forecast errors arising predominantly from uncertainties in the initial condition rather than deficiencies in the model. For precipitation assimilation, this assumption must be re-examined since rainfall in global models is based on parameterized moist physics with simplifying approximations, which can have significant systematic errors (Randall et al. 2003). The fact that analyses in the tropics are sensitive to the treatment of diabatic processes (Trenberth and Olson 1988) suggests that the influence of model errors are significant, if not dominant, in regions where observations are relatively sparse.

Since systematic model errors can lead to poor forecasts even with perfect initial conditions, the influence of model errors needs to be considered in order to assimilate precipitation information effectively in the presence of model biases. Typically, errors in physical parameterizations are projected as statedependent systematic errors in forecast tendencies, which are difficult to quantify without dense observation networks. The challenge of developing assimilation algorithms to account for systematic model errors is very much hindered by the lack of a priori knowledge of the nature of these errors. In the coming years more microwave rainfall data will become available from operational and research satellites, culminating to a constellation of 8 or more satellites to provide global rain measurements every 3 hours with the proposed Global Precipitation Measurement (GPM) mission in 2007. The ability to assimilate rainfall information effectively in the presence of forecast model biases will be crucial for realizing the full benefit from these observations.

This study explores an alternative rainfall assimilation scheme using forecast tendency corrections as the control variable within the general framework of variational continuous assimilation (VCA, Derber 
1989) to compensate for model deficiencies. Since model-predicted precipitation is diagnostically linked to temperature and moisture tendencies associated with parameterized moist physics, a VCA-type of approach offers a natural framework for assimilating precipitation data using moisture and/or temperature tendencies as the control variable. To test the effectiveness of such a scheme, we have developed a " $1+1$ " dimensional (1+1D) variational algorithm to assimilate 6-h accumulated TMI and SSM/I tropical rainfall in the Goddard Earth Observing System (GEOS) global data assimilation system (DAS). The 1+1D designation refers to the forward model for precipitation being a 6-h integration of a column model of the GEOS moist physics with prescribed large-scale forcing from the full GEOS DAS.

In earlier studies, before quantitative error estimates for microwave rain retrievals were available, we had tested the 1+1D scheme with several simplifications: (i) The cost function consisted only of the observation term, (ii) the correction was applied only to the model moisture tendency, and (iii) the moisture tendency correction has a prescribed vertical structure mimicking the Jacobian of the 6-h rain accumulation to moisture perturbations (Hou et al. 2000,2001). Results showed that, even with these simplifications, assimilating TMI and SSM/I tropical rain rates together with total precipitable water (TPW) estimates using this procedure is effective in improving GEOS analyses and short-range forecasts. Notably, it improves not only precipitation and moisture fields but also related climate parameters such as clouds and atmospheric radiation fluxes, as verified against the top-of-the-atmosphere radiation measurements from Clouds and the Earth's Radiation Energy System (CERES) sensors and brightness temperatures for moisture-sensitive channels of High-resolution Infrared Radiation Sounder (HIRS) (Hou et al. 2001).

This paper describes the full implementation of the 1+1D VCA scheme using background and observation errors together with augmented control variables including both moisture and temperature tendency corrections. Our previous work assessed the impact of precipitation assimilation on the monthlymean $2^{\circ} \times 2.5^{\circ} \mathrm{GEOS}$ analyses and forecasts ( ibid), the present study focuses on the impact of assimilating 6-h TMI and SSM/I rain rates on $1^{\circ} \times 1^{\circ}$ GEOS-3 synoptic analyses and forecasts of two prominent hurricanes, Bonnie and Floyd, in the tropics. Section 2 outlines the general methodology. Section 3 discusses implementation details and data usage in the GEOS-3 DAS. Section 4 describes the Bonnie and Floyd assimilation experiments. Sections 5 and 6 examine the impact of precipitation assimilation on 
GEOS-3 analyses and forecasts, respectively. The results are summarized in Sec. 7.

\section{Methodology}

Variational algorithms for precipitation assimilation typically seek to minimize a functional that measures the misfit between the model-predicted rain and the observed rain with respect to a control variable to which the model rain is sensitive. The control variable may be the initial condition (as in a conventional 4D variational scheme), a model attribute (such as the forecast tendency in a VCA scheme), or some combination of both (e.g., Zupanski et al. 2002). Since analysis techniques are built upon the assumption that the underlying error statistics are random, unbiased, stationary, and normally distributed, it is crucial that biases in the forecast model be removed (Dee and Todling 2000). In the case of precipitation, errors in the model-predicted rain derived from parameterized physics are invariably statedependent and systematic. In this study, we examine a 1+1D VCA procedure to assimilate observed surface rain rates to estimate and correct for systematic errors in temperature and/or moisture tendencies within a 6-h assimilation cycle. The functional to be minimized is:

$$
J(\delta w)=(\delta w)^{T} \mathbf{Q}^{-1}(\delta w)+\left\{\log [H(\delta w)]-y^{o}\right\}^{T} \mathbf{R}^{-1}\left\{\log [H(\delta w)]-y^{o}\right\}
$$

where $y^{\circ}$ is the logarithm of the observed rain, and $H$ is the precipitation observation operator. (The logarithmic singularity is removed with a minimal threshold value of $0.01 \mathrm{~mm} \mathrm{~d}^{-1}$.) $\mathbf{R}$ is the "observation" error covariance, which includes non-systematic errors in both observations and the forward model. Since precipitation is a non-negative quantity, a logarithmic transformation is used so that the observation error in terms of the relative error defined as $\varepsilon^{o}=y^{o} / y^{t}$ (where $y^{t}$ is the true rain rate) has an approximately lognormal distribution. Note that with this transformation, for an unbiased observation, $<\varepsilon^{p}>=1$, and $R_{i j}$ $=\log \left\langle\varepsilon_{i}^{0} \varepsilon_{j}^{0}\right\rangle$, where $i$ and $j$ are row and column indices (Cohn 1998). The control variable, $\delta w$, which is held constant within a 6 -h window, consists of a temperature tendency correction, $\delta T$, and a moisture tendency correction on the pseudo-relative humidity, $\delta q / q_{s}^{b}$, where $q_{s}^{b}$ is the background saturation specific 
humidity and the background can be a forecast or a pre-analysis (Sec. 3). The vector dimension of $\delta w$ is $2 \mathrm{~N}$, where $\mathrm{N}$ is the number of vertical model levels. $\mathbf{Q}$ is the error covariance for a prior estimate of $\delta w$.

For assimilating 6-h rain accumulation averaged over a model grid, the model rain is estimated from a 6-h integration of a nonlinear column model of GEOS-3 moist physics, $M$, as a function of the state vector. of temperature and specific humidity, $x=(T, q)$ :

$$
\partial x=M(x)+F+\delta x
$$

where $\partial_{t}$ denotes the partial derivative with respect to time, $F$ is the net tendency due to processes other than moist physics prescribed from a 3-h assimilation by the full GEOS-3 DAS from the beginning of the analysis cycle (See Hou el al. 2000 for details). Included in $F$ are contributions from dynamics, turbulence, radiation, and incremental analysis update (IAU) forcing due to all observations except precipitation. The observation operator for surface rain, $H$ is given by:

$$
H(\delta w)=-\int_{0}^{\Delta \tau} \int_{0}^{h} \rho \partial_{t} q(\delta w) d z d t, \quad \text { for } \partial_{q} q<0
$$

where $\partial_{q}$ is the moisture tendency in specific humidity, $\Delta \tau$ is the width of the analysis window, and $h$ is the model top. The forward model is constructed for time-accumulated rainfall rather than instantaneous rain rates since moist convection schemes used in global models are parameterized to represent ensemble averages of cloud and precipitation over a convective life cycle, dictating that some temporal and spatial averaging of the instantaneous observations at satellite footprint scales is necessary for physical consistency.

Consistent with the use of a column model for surface precipitation, rain rates are assumed to be uncorrelated horizontally, in which case $\mathbf{R}$ reduces to a scalar, $R$, given by the variance of relative observation errors in retrieved rain rates (assuming negligible random errors in the forward model): viz., $R$ $=\log \left[1+\left(\sigma^{\circ}\right)^{2}\right]$, where $\sigma^{\circ}$ is the error standard deviation given by $<\left(\varepsilon^{0}-1\right)^{2}>^{1 / 2}$ for rain rates greater than 0.1 $\mathrm{mm} \mathrm{h}^{-1}$. Bauer et al. (2002) estimated that the error standard deviation of instantaneous TMI retrievals averaged to $60 \mathrm{~km}$ grids ranges from $20-50 \%$ at low rain rates $\left(0.1 \mathrm{~mm} \mathrm{~h}^{-1}\right)$ to $5-20 \%$ at high rain rates 
$\left(20 \mathrm{~mm} \mathrm{~h}^{-1}\right)$. For $100 \mathrm{~km}$ averages, the errors are estimated to be less than $30 \%$ at low rain rates and $10 \%$ at high rain rates. Taking into account of additional errors arising from undersampling by TRMM overpasses in a 6-h interval on the order of $20-60 \%$ (Bell et al. 1990), $\sigma$ is assumed to be $15-50 \%$ for 6-h TMI rain averaged to $1^{\circ}$ latitude by $1^{\circ}$ longitude grids. In this study $\sigma^{\circ}$ is taken to be 0.3 for rain rates greater than $0.1 \mathrm{~mm} \mathrm{~h}^{-1}$, below which $\sigma^{\circ}$ is set to 0.7 , reflecting greater uncertainties in relative errors at very low rain rates. These values are by no means definitive but provide a reasonable starting point. Given the uncertainty in these estimates, the same $\sigma^{\circ}$ values are used for SSM/I rain rates. Even though the TMI retrievals are expected to be of higher quality due to its lower orbit, the differences between TMI and SSM/I are likely smaller than uncertainties in the intrinsic errors in each (William Olson, personal communication).

The model error covariance matrix, $\mathbf{Q}$, for $\delta w$ is parameterized in terms of the background temperature and pseudo-relative humidity errors based on the statistics of observation-minus-forecast $(\mathrm{O}-$ F) residuals, with the assumption that the errors are uncorrelated in the vertical and between the variables. The transformation of the moisture variable from specific humidity to pseudo-relative humidity renders $\mathbf{Q}$ implicitly a function of temperature through the saturation humidity and more homogeneous in space and time (Dee and da Silva 2003).

The solution to (1) is obtained by minimizing $J(\delta w)$ with respect to $\delta w$ using a quasi-Newton method. For a nonlinear observation operation, the gradient of $J$ must be computed at each iteration. An advantage of the limited dimension of a column model is that the gradient of $J$ may be computed numerically using a standard perturbation method. At each iteration, the vertical structure of $\delta w$ is determined by $\mathbf{Q}$ and the Jacobian of $H$. With $\mathbf{Q}$ consisting of only diagonal elements, the Hessian is positive definite, which guarantees convergence.

Compared with other techniques, the 1+1D VCA scheme differs from nudging or physical initialization in that it is a statistical analysis within the optimal estimation framework, even though they all explicitly modify the prognostic equations. As implemented in the GEOS DAS, the VCA scheme effectively operates as an online model bias estimation and correction for precipitation and moisture every 6 hours, similar in principle to the bias correction procedure described in Dee and Todling (2000). The main 
differences are that in our case the bias estimation and correction are carried out throughout the model integration and that there is no a priori assumption that systematic errors in moisture tendency are slowly varying (for a fast process such as precipitation). A well-known limitation of many conventional analysis schemes is that rainfall data can not be assimilated at locations where the background rain is zero, leading to the underutilizing the available information. By contrast, through correction of biases in the background rain, the VCA scheme can overcome this limitation to make effective use of observations. In its generalization to four dimensions, the VCA scheme is similar to 4DVAR schemes that employ the forecast model as a weak constraint. As a technique for precipitation analysis, the VCA scheme differs from other schemes in that the VCA-based precipitation estimate is not a forecast product but is determined by the 6-h rain accumulation from a continuous $4 \mathrm{D}$ data assimilation constrained by precipitation observations.

\section{Interface with GEOS-3 DAS and data usage}

The 1+1D VCA procedure may be implemented as an online bias estimation/correction scheme to improve the first-guess in an intermittent data assimilation system. But, in the GEOS-3 DAS, which uses an IAU scheme to distribute the influence of analysis increments as a constant forcing over the analysis time window (Bloom et al. 1996), it is natural to use the VCA scheme to estimate model tendency corrections based on precipitation data to be added to the IAU forcing due to other data types. This is accomplished by performing the $1+1 \mathrm{D}$ precipitation assimilation after a pre-analysis using the standard Physical-space Statistical Analysis System(PSAS, Cohn et al. 1998), which consists of a mutivariate analysis of wind, height, and surface pressure, and a univariate analysis of moisture using conventional observations, SSM/I surface wind speed and TPW data, and online temperature and moisture retrievals from TIROS Operational Vertical Sounder. This pre-analysis provides the large-scale forcing and IAU tendencies for the column model (2) and the background rain for quality control decisions.

A distinctive feature of the GEOS-DAS is that the IAU scheme virtually eliminates any spin-up in the precipitation and evaporation in assimilation cycles (Schubert et al. 1993). The GEOS-3 DAS is an upgraded version of the GEOS system that uses an interactive mosaic-type land surface model (Koster and 
Suarez 1993) and online temperature and moisture retrievals using TOVS radiances. The model rain consists of convective precipitation generated by the relaxed Arakawa-Schubert scheme with a Kesslertype re-evaporation of falling rain and large-scale precipitation from supersaturation.

In the GEOS-3 DAS, precipitation assimilation is implemented with the constraint that the column moisture does not depart by more than a few percent from the pre-analyzed TPW, which includes contributions from SSM/I data. This is accomplished by solving $(1)$ with the additional term: $\left(\sigma^{I P W}\right)^{-2}(\delta Z)^{2}$, where,

$$
\delta Z=\int_{0}^{\Delta \tau} \int_{0}^{h} \delta q(z) d z d t
$$

and $\sigma^{T P W}=0.05$. The quasi-Newton minimization algorithm typically converges between 5 to 25 iterations.

\section{a. Sensitivity to temperature tendency adjustment}

The general formulation of the VCA scheme uses for control variables tendency corrections of temperature and moisture, the input parameters for updating the moist physics at every time step. The corrections at each vertical level as given by (1) depend on the model error variance and the sensitivity of the observation operator for $6-\mathrm{h}$ rain to the control variables. Since temperature analyses are typically more accurate than moisture analyses, the assumption that $\mathbf{Q}$ follows the error variances of the background state implies that the cost function is less responsive to $\delta T$ than $\delta q$, unless the moist physics is more sensitive to temperature perturbations. Earlier studies have shown that moisture tendency corrections alone are effective for assimilating surface rain rates (e.g., Hou et al. 2001). Here we investigate the impact of extending the control variable to include temperature tendency corrections.

We performed three parallel offline experiments with (i) both moisture and temperature tendency corrections, (ii) moisture tendency correction only, and (iii) temperature tendency correction only. Figure 1 shows that the solution to (1) is virtually the same with or without temperature tendency corrections, which are negligible, as seen in Fig. 1b. Minimizing (1) using only temperature tendency corrections did not reduce the misfit between the model rain and the observed rain: The minimization yields $0.404 \mathrm{~mm} \mathrm{~h}^{-1}$, 
virtually unchanged from the background value of $0.408 \mathrm{~mm} \mathrm{~h}^{-1}$, compared with the observed rain of $0.0854 \mathrm{~mm} \mathrm{~h}^{-1}$. Artificially inflating the model error to 2.5 times the background error standard deviation to allow the analysis to draw closer to data leads to $0.400 \mathrm{~mm} \mathrm{~d}^{-1}$, which confirms the insensitivity of(1) to $\delta T$. Based on these results, only the moisture tendency correction is used in the remaining study, which effectively reduces the dimension of the control variable by half.

\section{b. Data usage}

The single footprint, instantaneous surface rain retrievals from the TMI and two SSM/I $(F 13$ and F14) sensors using the GPROF algorithm (Kummerow et al. 1996, Olson et al. 1996) are accumulated over 6 hours centered at analysis times $(0000,0600,1200,1800 \mathrm{UTC})$ and averaged to $1^{\circ} \mathrm{x} 1^{\circ} \mathrm{GEOS}-3$ grids. As microwave retrievals over land are less reliable than those over oceans, we restrict the data usage to oceanic areas within $30^{\circ} \mathrm{N}$ and $30^{\circ} \mathrm{S}$, subject to two online quality control (QC) checks based on the observation-minus-background $(\mathrm{O}-\mathrm{B})$ residuals in 6-h rain accumulation, where the background rain is obtained from (3) with $\delta w=0$. First, a gross check is applied to eliminate outliers with O-B residuals greater than $5 \mathrm{~mm} \mathrm{~h}^{-1}$, as the minimization procedure would fail for excessively large O-B values. Data rejected by this criterion typically account for less than 0.5 percent of all valid observations. Second, no minimization is performed unless the $\mathrm{O}-\mathrm{B}$ residual exceeds $1 \mathrm{~mm}$ in 6 hours (i.e., $0.17 \mathrm{~mm} \mathrm{~h}^{-1}$ ), so that data are ingested only when the background is appreciably different from the observed. Since low rain rates account for much of the observed and model precipitation, this minimum threshold greatly reduces the computational cost without compromising the quality of the analysis.

Since the 6-h precipitation observation operator is nonlinear, it is possible for the minimization procedure to produce excessively large moisture tendency corrections for a moderate change in rain rate. An after-analysis QC check is applied to the solution to (1) to prevent the temperature and humidity at the end of the 6-h column model integration, $x^{6 h}$, from deviating from the background values by more than a pre-determined amount. For moisture, the cutoff value is 2 standard deviations of the tropical-mean O-F bias in specific humidity in negative $\mathrm{O}-\mathrm{B}$ cases and 3 standard deviations in positive $\mathrm{O}-\mathrm{B}$ cases. These 
cutoff values are obtained empirically from online assimilation experiments based on RMS error reductions in daily tropical precipitation (relative to TMI and SSM/I rain rates) and all-sky outgoing longwave radiation (against CERES measurements). The larger threshold value for positive $\mathrm{O}-\mathrm{B}$ cases reflects that the VCA scheme is more effective in reducing than enhancing the background rain. Typically, $20 \%$ of the number of minimizations performed are rejected by this afer-analysis check.

The data usage at a single analysis time is illustrated in Table 1 for 1200 UTC 20 August 1998. Of 10,480 valid TMI and SSM/I tropical observations averaged to $1^{\circ} \times 1^{\circ}$ model grids, the $\mathrm{O}-\mathrm{B}$ residual is non-zero at 7887 locations, including 5545 locations at which the model rain is within $0.17 \mathrm{~mm} \mathrm{~h}^{-1}$ of the observed value and no minimization is performed. Of the 2310 minimization solutions, 1787 are accepted by the after-analysis QC check. Altogether, 555 of the 7887 observations (roughly 7\%) are rejected by the two QC checks. Table 1 also confirms that the VCA scheme is effective in assimilating rain rates at locations where there is no background rain. However, the benefits in the GEOS-3 DAS are limited by that the background rain is zero at only $10 \%$ of all locations with observed rain.

Figure 2 compares the background rain and the analyzed column model rain given by (1) against observations. In Fig. 2a are the background rain plotted against observations at 1072 'raining' grid locations at which the minimization was performed - excluded those that had no rain in either the background or the observation. Figure $2 \mathrm{~b}$ shows the 944 analyzed rain rates accepted by the after-analysis QC check lying predominantly along the diagonal against observations, reflecting the smaller observationminus-analysis (O-A) residuals than the corresponding O-B residuals in Fig. 2a. This shows that the continuous application of moisture tendency corrections over $6 \mathrm{~h}$ is effective in overcoming systematic errors in the column model to produce analyzed rain rates close to observations. The notable exception is the scatter at observed rain rates below $0.1 \mathrm{~mm} \mathrm{~h}^{-1}$, which is a direct result of the larger errors assigned to these low rain rates (See Sec. 2) to prevent the minimization solution from over-fitting that data. The distinct transition in the character of analyzed rain rates in Fig. $2 \mathrm{~b}$ suggests that the minimization solution is indeed responsive to observation error specification and that rainfall data usage can be improved as better quantitative error estimates become available. 


\section{Bonnie and Floyd assimilation experiments}

A prominent atmospheric feature difficult to capture accurately in current global models and analyses is the tropical cyclone system with fine structures requiring a resolution better than one-sixth of a degree to adequately resolve (Kurihara et al.1990). Yet, an accurate depiction of such systems in analyses is crucial for providing realistic initial conditions for numerical weather forecasts and boundary forcing for nested regional models. Since intense rainfall are associated with hurricane events, precipitation assimilation may be expected to directly affect the representation of hurricanes in global systems. In this study we examine how assimilating tropical TMI and SSM/I surface rain rates over oceans affects $1^{\circ} \times 1^{\circ} \mathrm{GEOS}-3$ analyses and forecasts of two distinctly different Atlantic storm systems - Bonnie and Floyd - from the 1998-1999 period. As a tropical storm, Bonnie was characterized by a notably asymmetric vortex with a wind maximum to the northeast of the center, while Floyd was much less asymmetric in its early stages of development. For each storm, we performed two parallel assimilation experiments - one(PRECIP) with and one (CNTRL) without TMI and SSM/I rain rates - for a two-week period centered around the time when the system became a hurricane.

Bonnie reached tropical storm intensity at 1200 UTC 20 August 1998 and subsequently became a hurricane with a nearly complete eyewall by 0000 UTC 22 August (Avila 1998). It eventually made landfall near Wilmington, North Carolina on 27 August. The assimilation experiments for Bonnie extend from 15 August to 29 August. Floyd can be traced to a tropical depression east of the Lesser Antilles on 7 September 1999. The system intensified to a tropical storm at 0600 UTC 8 September and upgraded to a hurricane by 1200 UTC 10 September near northen Leeward Islands (Pasch et al 1999). Floyd made landfall near Cape Fear, North Carolina on 16 September, then moved on to New England. The Floyd experiments extend from 3 September to 17 September.

\section{Impact on analyses}


Hurricane vortices in global analyses with limited spatial resolution are generally weak, overly smooth, and not as sharply defined compared with observations ( $\mathrm{Pu}$ and Braun 2001). Rainfall assimilation is not expected to overcome this inherent limitation in model resolution. This section examines the extent to which assimilating TMI and SSM/I surface rain rates may improve the synoptic features of Bonnie and Floyd in $1^{\circ} \times 1^{\circ}$ model GEOS-3 analyses.

\section{a. $\quad$ Track trajectory and intensity}

Figure 3 compares analyzed positions of the minimum surface pressure of Bonnie and Floyd at 12 h intervals with the 'best track' published by the Hurricane Research Division (HRD) of National Oceanic and Atmospheric Administration (NOAA). Each track traces the system from the tropical storm stage to the time just before landfall. The GEOS-3 tracks of Bonnie and Floyd, with or without rainfall data, are in general agreement with the best track to within the one-degree accuracy of analyzed positions, which are rendered to the closest integer degree in latitude and longitude. There are instances that rainfall assimilation improves the storm position by more than one degree, as for Bonnie at 1200 UTC 20 August 1998.

The along-track sea-level pressure (SLP) minimum and $850 \mathrm{hPa}$ vorticity are shown in Fig. 4. As noted earlier, hurricanes in global analyses are typically weak. In the early stages when Bonnie and Floyd are tropical storms, the minimum pressures in the CNTRL analyses are within $5 \mathrm{hPa}$ of the best track values. But as the storms intensified to hurricane strengths, the limited resolution of $1^{\circ} \times 1^{\circ}$ analyses cannot capture the rapid surface pressure deepening seen in point observations (Bonnie reached $954 \mathrm{hPa}$ at 0000 UTC 24 August 1998, and Floyd reached $921 \mathrm{hPa}$ at 1200 UTC 13 September 1999). Figure 4 shows that rainfall assimilation yields slightly lower pressure minima and enhanced low-level cyclonic vorticity, suggestive of marginal improvements in the storm intensity throughout the hurricane stage. However, rainfall assimilation maynot always lead to a lower sea-level pressure minimum or stronger low-level vorticity for a variety of reasons. For one, its impact may be to relocate the storm center instead of strengthening a correctly-positioned storm, as in the case of Bonnie at 1200UTC 20 August 1998. Also, assimilation of rain rates from a partial satellite coverage that misses a major section of the storm (as during the early 
stages of Tropical Storm Floyd) can lead to aliasing problems by modifying the latent heating only in observed portion of the storm, thus misrepresenting the circulation pattern. These issues will require detailed analyses beyond the scope of this study.

\section{b. $\quad$ Precipitation and sea-level pressure}

The impact of assimilating TMI and SSM/Irain rates on rainfall analyses is manifest in Fig. 5, which shows reduced daily-mean root-mean-square (rms) errors and improved spatial correlations within a $20^{\circ}$ $\mathrm{x} 30^{\circ}$ latitude-longitude moving domain along the storm tracks. The one exception is the result for 26 August, at which time Bonnie was entirely north of $30^{\circ} \mathrm{N}$, beyond the domain for rainfall assimilation in these experiments. The results show an overall improvement in both the intensity and spatial pattern of precipitation associated with the storm system. For rain rates averaged over the entire tropics for the 2week assimilation period, the improvements are even greater. For example, in the case of Bonnie, rainfall assimilation yields a bias reduction of $61 \%$ and a smaller error standard deviation by $33 \%$, averaged over the entire tropics.

Illustrated in Fig. 6 are improved rainfall intensity and structure for Floyd at 1200 UTC 10 September 1999. The storm center in the CNTRL analysis coincides with the best track position and remains unchanged with the addition of rainfall data. Relative to TMI and SSM/I observations, the rms error of the CNTRL rainfall analysis is $1.03 \mathrm{~mm} \mathrm{~h}^{-1}$ over the domain shown in Fig. 6. Rainfall assimilation reduces the rms error in the PRECIP analysis by $21 \%$ and increases the spatial correlation from 0.14 to 0.64 . The VCA algorithm is effective in removing the spurious rainfall centered around $66^{\circ} \mathrm{W}$ and $19^{\circ} \mathrm{N}$ in the CNTRL (Fig. 6b) and enhancing precipitation in the east and south quadrants of the hurricane vortex (Fig. 6c), as observed. Since precipitation assimilation directly alters the vertical motion field, the improved precipitation within the IAU analysis framework can have a substantial impact on the large-scale circulation, as shown in Fig. 6d. Averaged over the domain shown in the figure, the spatial correlation between changes in the surface rain field and the $500 \mathrm{hPa}$ omega velocity is -0.71 . Also evident are the increased horizontal divergence at $200 \mathrm{hPa}$ over the center of the storm and enhanced subsidence at $500 \mathrm{hPa}$ in the surrounding 
areas away from the hurricane vortex. A more realistic subsidence environment not only promotes a more confined hurricane structure but is also crucial for preventing the low-level moisture from precipitating outside the storm, which is a common problem in global systems (as in the CNTRL). Typically, rainfall assimilation can modify the horizontal divergent winds at $200 \mathrm{hPa}$ by as much as $10 \mathrm{~m} \mathrm{~s}^{-1}$, which can have a large impact on short-range forecasts, which will be examined in Sec. 5.

\section{c. Storm structures}

Figure 7a shows CNTRL analyses of the $850 \mathrm{hPa}$ geopotential height and wind speed for Bonnie at 1200 UTC 21 August, 12 hours before Bonnie gained hurricane strength. The storm center inferred from the minimum wind speed is in agreement, within the resolution uncertainty, with the best track position of $19.5^{\circ} \mathrm{N}$ and $64.5^{\circ} \mathrm{W}$. But the system is too weak with overly broad features compared with observations (Avila 1998). The same quantities from the PRECIP analysis are shown in Fig. 7b, superimposed with the geopotential anomalybetween the two analyses, which is negative at the storm center and positive to the east and west, corresponding to a deeper and more contracted system with increased horizontal winds to the north of the storm center. This pronounced asymmetry is consistent with wind observations from ships and high-resolution low-cloud wind analysis (Avila 1998). Changes in these fields at other levels indicate an overall intensification of the storm in the lower troposphere and a slight weakening above the mid-troposphere, leading to a more realistic structure typical of tropical storm systems. These changes are evident in the vertical structure of a meridional cross-section through the center of Bonnie shown in Figs. $8 \mathrm{a}$ and $8 \mathrm{~b}$. Compared with the CNTRL, the PRECIP analysis shows a better defined vortex structure with a distinct eye-like feature (identified by a column of low wind speed), a more pronounced warm-core in the mid-troposphere, stronger low-level winds to the north of the center, and an enhanced low-level vorticity maximum (by more than $40 \%$ ), all features characteristic of hurricane structures inferred from in-situ observations (e.g., Anthes 1982).

Hurricane vortices in global models are generally too weak at the low levels yet too strong at the upper levels, leading to an incorrectly placed wind maximum in the analysis compared with observations. 
Rainfall assimilation using the VCA procedure strengthens the low-level winds while weakening winds between $600 \mathrm{hPa}$ and $300 \mathrm{hPa}$, as can be seen in Fig. 8 although it is more evident in the zonal cross section (not shown). This downward displacement of the wind maximum (and the kinetic energymaximum) is similar to what is often achieved by imposing an artificial "bogus" vortex to improve hurricanes in analyses (e.g. Lord 1990). However, the bogusing technique has several limitations: It can be applied only to mature hurricanes; it does not perform well for highly asymmetric storm systems; and the specification of a moisture field consistent with the imposed vortex is a problem ( $\mathrm{Pu}$ and Braun 2001). Precipitation assimilation offers an altemative to bogusing in a way that is more consistent with model physics and applicable in early stages of storm development regardless of the azimuthal asymmetry. However, the benefit of rainfall assimilation is currently limited by model resolutions. But, as models continue to evolve to finer resolutions, precipitation assimilation has the potential to be the method of choice for improving the realism of storms in analyses and early warning capabilities.

In the case of Floyd, the improvements are even more evident. Figures $7 \mathrm{c}$ and $7 \mathrm{~d}$ show the 850 hPa geopotential height and wind speed at 0000 UTC 11 September 1999, shortly after Floyd reached hurricane strength. The PRECIP analysis shows a substantially stronger storm, with a clearly defined center coinciding with the best track position at $20.8^{\circ} \mathrm{N}$ and $60.4^{\circ} \mathrm{W}$. The $850 \mathrm{hPa}$ height anomaly is negative to the north of the storm center in the CNTRL, signifying a deepening and a northward shift. Figures $8 \mathrm{c}$ and $8 \mathrm{~d}$ show a near doubling in the strength of the low-level vorticity, a downward displacement of an enhanced low-level wind maxima, a pronounced warm core, and a well-defined eye-like feature in the column of low-speed winds: The impact of these improved structures on forecast is examined in the next section.

\section{Impact on forecasts}

Results in the previous section showed that rainfall assimilation can significantly modify moisture, wind, and temperature analyses. To assess whether these changes in analyses are, in fact, improvements, we performed parallel Bonnie and Floyd forecasts initialized with PRECIP and CNTRL analyses, with and without rainfall data, respectively. The initial conditions are from the early stages of hurricane formation starting with the first analysis level with a well-formed inner core namely, 1200 UTC 20 August 1998 for 
Bonnie and 1200 UTC 10 September 1999 for Floyd (Pasch et al. 1999). The forecasts were issued 12 $\mathrm{h}$ apart over a two-day period.

Comparisons of storm track forecasts with the NOAA best track analysis are shown in Fig. 9 for Bonnie and Fig. 10 for Floyd. The impact ranges from positive to near-neutral. Hurricanes in forecasts initialized with CNTRL analyses consistently move too fast along paths that are displaced eastward and northward relative to the best track. In the best cases (Figs. 9a and 10b), forecasts initialized with PRECIP analyses show significant reductions in both spatial and temporal displacement errors. These tend to be associated with initial conditions with significant improvements in both the storm intensity and position. In other cases, forecast improvements reflect mostly reduced errors in spatial locations rather than temporal displacements, as in Figs. 9b and 10a. Other forecast experiments show improvements that fall within the ranges shown in Figs. 9 and 10.

To verify that the improvements shown in Figs. 9 and 10 result directly from assimilating rainfall information in the initial condition, we varied the assigned weighting for the TMI and SSM/I rain rates in the VCA procedure to investigate how the resulting analyses affect storm forecasts. In addition to CNTRL and PRECIP analyses (for which the precipitation observation error standard deviation, $\sigma_{o}$, is effectively infinity and $30 \%$, respectively), we obtained two more PRECIP analyses with $\sigma_{o}=80 \%$ and $250 \%$ and performed additional forecasts using them as initial conditions. Results for the 5-day Bonnie forecast issued from 1200 UTC20 August 1998 are shown in Figs. 11. As the initial analysis is weighted more towards the TMI and SSM/I rain rates, there is a systematic improvement in the track forecast (Fig. 11a) and an overall increase in the spatial correlation between the 5-day precipitation forecast and observations (Fig. 11b). The exception is the rain forecast within the first 24 hours, in which case the forecast differences are likely too small to overcome measurement and sampling uncertainties in rainfall observations. However, the overall results show a systematic increase in forecast skills as more rainfall information is retained in the initial state, suggesting that the improvements are directly attributable to the use of rainfall data in the initial condition.

Shown in Fig. 12 are two examples of the rms errors and spatial correlations in 5-day precipitation forecasts issued from analyses with and without TMI and SSM/I rain rates. The Bonnie forecast (Figs. 12a 
and 12c) was issued from 1200 UTC 20 August 1998. The Floyd forecast (Fig. 12b and 12d) was initialized from 0000 UTC 11 September 1999. In each case, the statistics are for a $20^{\circ}$ latitude by $30^{\circ}$ longitude moving domain along the analyzed storm track. Results show a general reduction in rms errors and a marked increase in spatial correlations. These improvements are also apparent in terms of the $\ldots$ improved threat scores for different rain thresholds commonly used to assess quantitative precipitation forecast skills (QPFs, Wilks 1995).

\section{Summary and discussion}

We have described a 1+1D VCA algorithm for assimilating surface precipitation using the forecast model as a weak constraint and examined its effectiveness in using 6-h TMI and SSM/I tropical rain rates to improve GEOS-3 analysis and forecasts. In its full implementation the scheme uses temperature and moisture tendency corrections to compensate for model deficiencies. But the observation operator for 6-h rain accumulation proved to be insensitive to temperature tendency correction, and moisture tendency correction alone made an effective control variable since precipitation in models is diagnostically linked to the time-rate-of-change of atmospheric moisture.

In earlier studies, we have shown that assimilating TMI and SSM/I rain rates improves precipitation and related climate parameters such as radiative fluxes and cloud forcing in the GEOS analysis. This study focused on the impact of rainfall assimilation on $1^{\circ} \times 1^{\circ}$ GEOS-3 analyses and forecasts of synoptic features of prominent tropical weather systems using Hurricanes Bonnie and Floyd as case studies. For each storm, we performed two parallel assimilation experiments with and without rainfall data. Results show that assimilating TMI and SSM/I surface rain rates using the 1+1D moisture-tendency-correction VCA scheme yields more realistic storm structures through improved precipitation analysis. Forecast experiments show that the improved analyses provide better initial conditions for 5-day track and precipitation forecasts for Bonnie and Floyd. The improved forecast skills have been substantiated in a sensitivity study that showed a systematic increase in forecast skills as more rainfall information was retained in the initial condition. The 
improved forecasts provide evidence that the VCA scheme is capable of using rainfall data to improve analyses of prognostic model variables used to initialize the forecasts.

Results of this study suggest that addressing model deficiencies in moisture tendency may be crucial to making effective use of precipitation observations in data assimilation. Moreover, since precipitation is a model-diagnosed quantity, it affects forecasts only through its influence on model prognostic variables. During an assimilation cycle, the full benefit of precipitation data can be realized only if analyses of wind, temperature, moisture, and pressure are allowed to respond to an improved rainfall analysis. In the GEOS$3 \mathrm{DAS}$, the prognostic model variables continually adjust to changes in precipitation and the associated latent heating over a 6-h assimilation window, in a manner similar to dynamic initialization as discussed in Sec. 3 . In our work thus far, the 1+1D VCA scheme has been shown to be effective for assimilating rainfall data in the tropics, where the model precipitation is known to be sensitive to parameterized moist physics in a vertical column. The effectiveness of such a column model approach to rainfall assimilation in the extratropics, where atmospheric processes are governed by multivariate quasigeostrophic dynamics, will require further investigation.

As a technique for rainfall assimilation, the 1+1D VCA scheme differs from nudging or physical initialization in that it is a statistical analysis within the optimal estimation framework, even though they all modify the model's prognostic tendencies. As implemented in the GEOSDAS, the VCA scheme effectively operates as an online model bias estimation and correction for precipitation and moisture every 6 hours. As such, the scheme is capable of assimilating precipitation observations at locations where the background rain is zero. More generally, VCA tendency corrections may be included as a part of an augmented control variable within the 4DVAR framework or as a model error estimator in sequential analysis schemes.

Acknowledgments. It is a pleasure to acknowledge the support of this work by Dr. Ramesh Kakar, the TRMM Program Scientist, through NASA Grant 621-15. 
Table 1. Data usage at tropical TMI+SSM/I observation locations at 1200 UTC 20 August 1998

\begin{tabular}{|l|l|l|l|l|l|}
\hline Observation & Background & $\begin{array}{l}\text { Number of } \\
\text { Observations }\end{array}$ & $\begin{array}{l}|\mathrm{O}-\mathrm{B}|>0.17 \\
\mathrm{~mm} \mathrm{~h}^{-1}\end{array}$ & $\begin{array}{l}|\mathrm{O}-\mathrm{B}|<5.0 \\
\mathrm{~mm} \mathrm{~h}^{-1}\end{array}$ & $\begin{array}{l}\text { Accepted by }\left|q^{6 h}-q^{b}\right| \\
\text { QC check }\end{array}$ \\
\hline Rain & Rain & 1905 & 1102 & 1072 & 944 \\
\hline Rain & No Rain & 201 & 58 & 56 & 47 \\
\hline No Rain & Rain & 5781 & 1182 & 1182 & 796 \\
\hline Subtotal & 7887 & 2342 & 2310 & 1787 \\
\hline \multicolumn{7}{|l}{ Total number of $1^{\circ} \times 1^{\circ}$ observations: $10,480$. Non-raining grids in both observations and the model: 2593} \\
\hline
\end{tabular}




\section{REFERENCES}

Anthes, R., 1982: Tropical Cyclones. Their evolution, structure and effects. Amer. Meteor. Soc., 208 pp.

Avila, L., cited 1998: Preliminary report. Hurricane Bonnie, 19-30 August 1998. National Hurricane Center, 11691 S.W. 17th Street, Miami, FL, 33165-2149. 10 pp. [Available online from http://www.nhc.noaa.gov/1998bonnie.html].

Bauer, P., J.-F. Mahfouf, W. S. Olson, F. S. Marzano, S. di Michele, A. Tassa, and A. Mugnai, 2002: Error analysis of TMI rainfall estimates over ocean for variational data assimilation. Q. J. Roy. Meteor. Soc., 128, 2129-2144.

Bell, T. L., A. Abdullah, R. Martin, and G. North, 1990: Sampling errors for satellite-derived tropical rainfall: Monte Carlo study using a space-time stochastic model.J. Geophys. Res., 95, 2195-2205.

Bloom, S.C., L.L. Takacs, A.M. da Silva, and D.V. Ledvina, 1996: Data assimilation using incremental analysis updates. Mon. Wea. Rev., 124, 1256-1271.

Cohn, S., 1998: An introduction to estimation theory. J. Meteor. Soc. Japan, 75, 257-288.

-----, A.da Silva, J. Guo, M. Sienkiewicz, and D. Limbic, 1998: Assessing the effect of data selection with the DAO Physical-space Statistical Analysis System. Mon. Wea. Rev., 126, 2913-2926.

Dee, D.P., and R. Todling, 2000: Data assimilation in the presence of forecast bias: The GEOS moisture analysis. Mon. Wea. Rev., 128, 3268-3282.

-----, and A. M. da Silva, 2003: The choice of variable for atmospheric moisture analysis. Mon. Wea. Rev., 131, 151-171.

Derber, J.C., 1989: A variational continuous assimilation technique. Mon. Wea. Rev., 117, 2437-2446.

Hou, A.Y., D. Ledvina, A. da Silva, S. Zhang, J. Joiner, R. Atlas, G. Huffman, and C. Kummerow, 2000: Assimilation of SSM/I-derived surface rainfall and total precipitable water for improving the GEOS analysis for climate studies. Mon. Wea. Rev., 128, 509-537.

----, S. Zhang, A. da Silva, and W. Olson, 2000: Improving assimilated global datasets using TMI rainfall and columnar moisture observations, J. Climate, 13, 4180-4195. 
----, S. Zhang, A. da Silva, W. Olson, C. Kummerow, and J. Simpson, 2001: Improving global analysis and short-range forecast using rainfall and moisture observations derived from TRMM and SSM/I passive microwave sensors. Bull. Amer. Meteor. Soc., 81, 659-679.

Krishnamurti, T.N., H.S. Bedi, and K. Ingles, 1993: Physical initialization using SSM/I rain rates. Tellus, 45A, 247-269.

Kummerow, C., W. Olson, and L. Giglio, 1996: A simplified scheme for obtaining precipitation and vertical hydrometeor profiles from passive microwave sensors. IEEE Trans. Geosci. Remote Sensing, 34, 1213-1232.

Kurihara, Y., M. A. Bender, R. E. Tuleya, and R. Ross, 1990: Prediction experiments of Hurricane Gloria (1985) using a multiply nested movable mesh model. Mon. Wea. Rev., 118, 2185-2198.

Lord, S. J., 1991: A bogusing system for vortex circulations in the National Meteorological Center global forecast model. Preprints, 19th Conference on Hurricanes and Tropical Meteorology, Miami, FL, Amer. Meteor. Soc., 328-330.

Marecal, V., and J.-F. Mahfouf, 2002: Four-dimensional assimilation of total column water vapor in rainy areas. Mon. Wea. Rev., 130, 43-58.

Olson, W.S., C.D. Kummerow, G.M. Heymsfield, and L. Giglio, 1996: A method for combined passiveactive microwave retrievals of cloud and precipitation profiles. J. Appl. Meteor., 35,1763-1789.

Pasch, R. J., T. Kimberlain, and S. Stewart, cited 1999: Preliminary report. Hurricane Floyd, 7-17 September 1999. National Hurricane Center, 11691 S.W. 17th Street, Miami, FL, 33165-2149. $22 \mathrm{pp}$. [Available online from http://www.nhc.noaa.gov/1999floyd.html].

$\mathrm{Pu}, \mathrm{Z}$. H., and S. Braun, 2001: Evaluation of bogus vortex techniques with four-dimensional variational data assimilation. Mon. Wea. Rev., 129, 2023-2039.

Randall, D., S. Krueger, C. Bretherton, J. Curry, P. Duynkerke, M. Moncrieff, B. Ryan, D. Starr, M. Miller, W. Rossow, G. Tselioudis, B. Wielicki, 2003: Confronting models with data: The GEWEX Cloud Systems Study. Bull. Amer. Meteor. Soc., 84, 455-469.

Schubert, S., J. Pfaendtner, and R. Rood, 1993: An assimilated data set for Earth Science applications. Bull. Amer. Meteor. Soc., 74, 2331-2342. 
Treadon, R.E., 1997: Assimilation of Satellite Derived Precipitation Estimates within the NCEP GDAS. Ph.D thesis, Florida State University, Tallahasse, Florida. 348 pp.

Trenberth, K.E., and J.G. Olson, 1988: An evaluation and intercomparison of global analyses from the National Meteorological Center and the European Center for Medium Range Weather Forecasts. Bull. Amer. Meteor. Soc., 69, 1047-1057.

Tsuyuki, T., 1997: Variational data assimilation in the tropics using precipitation data. Part III: Assimilation of SSM/I precipitation rates. Mon. Wea. Rev., 125, 1447-1464.

Wilks, D. S., 1995: Statistical Methods in the Atmospheric Science. Academic Press. 467 pp.

Zupanski, M., D. Zupanski, D. Parrish, E, Rogers, and G. DiMego, 2002: Four-dimensional variational data assimilation for the blizzard of 2000. Mon. Wea. Rev., 130, 1967-1988. 
Figure Captions

Figure 1. Minimization solutions to (1) with and without temperature tendency correction. (a) Solid circles show the solution for moisture tendency correction with both temperature and moisture tendency corrections as the control variable. Open circles are for the case with only moisture tendency corrections. (b) Solution for temperature tendency correction with both temperature and moisture tendency corrections. The labels indicate the observed, background, and analyzed rain rates at this grid location.

Figure 2. (a) Background rain versus observations at 1200 UTC 20 August 1998. Shown are rain rates at1072 grid locations accepted by O-B QC checks for minimization. Not included are locations with no rain in either the background or the observation. (b) Analyzed rain rates given by (1) accepted by afteranalysis QC checks versus observations. The scatter at very low rain rates reflects the large error standard deviations assigned to observed rain rates below $0.1 \mathrm{~mm} \mathrm{~h}^{-1}$ (See Sec. 2).

Figure 3. Analyzed 12-h positions of the minimum surface pressure of Bonnie and Floyd (rendered to the closest integer degree in latitude and longitude) from CNTRL and PRECIP analyses, compared with NOAA best track locations. The first analysis time of the track is marked for each storm.

Figure 4. (a) Minimum sea-level pressure (SLP) in $\mathrm{mm} \mathrm{d}^{-1}$ along the track of Tropical Storm Bonnie (dotted lines) and Hurricane Bonnie (solid lines). The open circles show the CNTRL analysis and the crosses are the PRECIP analysis. (b) Same as (a) but for Floyd. (c) $850 \mathrm{hPa}$ vorticity in units of $10^{-4} \mathrm{~s}^{-1}$ along the Bonnie track. (d) Same as (c) except for Floyd.

Figure 5. (a) Root-mean-square (rms) errors and spatial correlation of daily averaged rain rate over a $20^{\circ}$ latitude and $30^{\circ}$ longitude moving domain along Bonnie's track. The black and white bars refer to CNTRL and PRECIP analyses, respectively. Combined TMI and SSM/I daily rain rates are used for verification. (b) Same as (a) except for Floyd. 
Figure 6. (a) Combined TMI and SSM/I observations of surface rain in $\mathrm{mm} \mathrm{h}^{-1}$ at $1200 \mathrm{UTC} 10$ September 1999. (b) Surface rain in $\mathrm{mm} \mathrm{h}^{-1}$ (shaded) and sea-level pressure in $\mathrm{hPa}$ (contours) in CNTRL at the same analysis time. (c) Same as (b) except for PRECIP analysis. (d) Changes in $500 \mathrm{hPa}$ omega velocity in $\mathrm{hPa} \mathrm{d}^{-1}$ (shaded, with negative values indicating rising motion) and horizontal winds in $\mathrm{m} \mathrm{s}^{-1}$ at $200 \mathrm{hPa}$ between PRECIP and CNTRL analyses. The vector scale for $10 \mathrm{~m} \mathrm{~s}^{-1}$ is given for reference.

Figure 7. Analyses of $850 \mathrm{hPa}$ geopotential height in $\mathrm{m}$ (thick line) and wind speed in $\mathrm{m} \mathrm{s}^{-1}$ (shaded). (a) CNTRL analysis at 1200 UTC 21 August 1998 for Bonnie. (b) Same as (a) but for PRECIP analysis. The thin contours show PRECIP minus CNTRL differences in $850 \mathrm{hPa}$ geopotential height. (c) CNTRL analysis for Floyd at 0000 UTC 11 September 1999. (d) Same as (c) but for PRECIP analyses with the thin lines showing $850 \mathrm{hPa}$ geopotential height differences between PRECIP and CNTRL.

Figure 8. Upper panels: Meridional-vertical cross-sections at $65^{\circ} \mathrm{W}$ through the center of Bonnie at 1200 UTC 21 August 1998. Shown are wind speed in $\mathrm{m} \mathrm{s}^{-1}$ (shaded), relative vorticity in $\mathrm{s}^{-1}$ (thick lines at intervals of $3 \times 10^{-5}$, with the zero contour omitted), and temperature in ${ }^{\circ} \mathrm{C}$ (thin lines). (a) CNTRL analysis. (b) PRECIP analysis. Lower panels: Similar cross-sections for Floyd at $60^{\circ} \mathrm{W}$ and 0000 UTC 11 September 1999. (c) CNTRL analysis. (d) PRECIP analysis.

Figure 9. Five-day Bonnie track forecasts. Triangles and squares mark forecasts (dashes) initialized with CNTRL and PRECIP analyses, respectively. The storm positions are rendered to the closest integer degree in latitude and longitude. The circles show positions of the best track (solid). (a) Forecasts issued from 1200 UTC 20 August 1998. (b) Forecasts issued from 1200 UTC 21 August 1998.

Figure 10. Five-day Floyd track forecasts. Triangles and squares mark forecasts (dashes) initialized with CNTRL and PRECIP analyses, respectively. The storm positions are rendered to the closest integer degree in latitude and longitude. The circles show positions of the best track (solid). (a) Forecasts initialized from 1200 UTC 10 September 1999. (b) Forecasts issued from 0000 UTC 11September 1999. 
Figure 11. Sensitivity of a 5-day Bonnie forecast from 1200 UTC 20 August 1998 to TMI and SSM/I rainfall information retained in the initial condition. (a) Comparison of 5-day track forecast with the NOAA best track analysis. (b) Spatial correlations between 5-day precipitation forecasts and combined TMI and SSM/I daily rain rates. The legend identifies the forecasts by the error standard deviation for rain $\left(\sigma_{o}\right)$ in the analysis used for initial condition.

Figure 12. Root-mean-squar (rms) errors and spatial correlations of 5-day precipitation forecasts initialized with CNTRL analysis (black) and PRECIP analysis (white). (a) Bonnie forecast issued from 1200 UTC 20 August 1998. (b) Floyd forecast issued from 0000 UTC 11 September 1999. The statistics are for a $20^{\circ}$ latitude by $30^{\circ}$ longitude moving domain along the analyzed storm track. QPF Threat Scores for the Day 3 precipitation forecast are shown in (c) and (d) for Bonnie and Floyd, respectively. A higher Threat Score indicates greater forecast skills. 

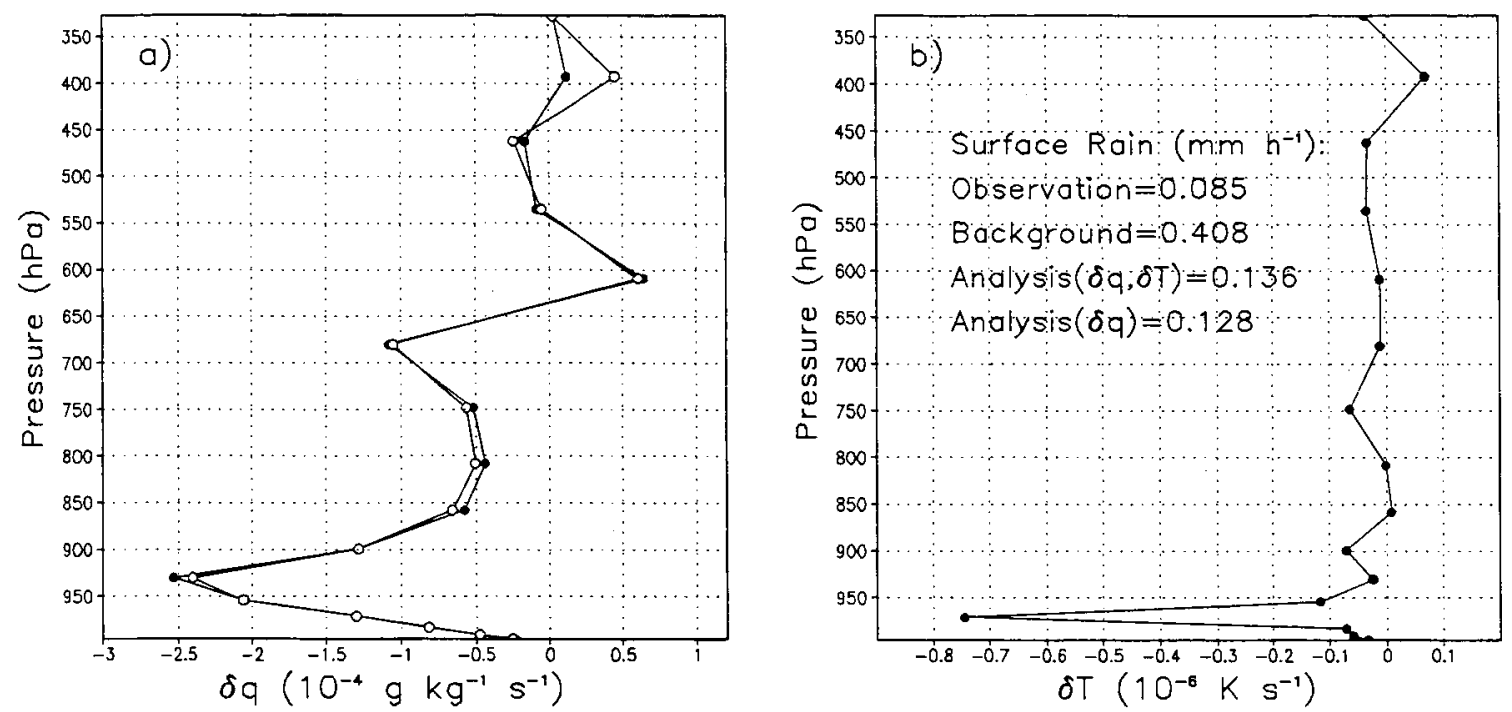

Figure 1. Minimization solutions to (1) with and without temperature tendency correction. (a) Solid circles show the solution for moisture tendency correction with both temperature and moisture tendency corrections as the control variable. Open circles are for the case with only moisture tendency corrections. (b) Solution for temperature tendency correction with both temperature and moisture tendency corrections. The labels indicate the observed, background, and analyzed rain rates at this grid location. 
a) Observation vs. Bockground

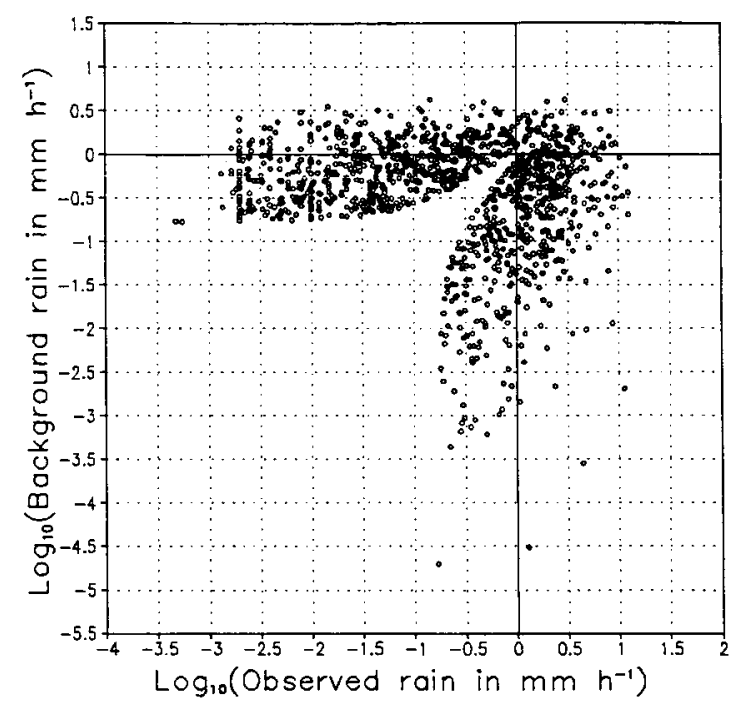

b) Observation vs. Analysis

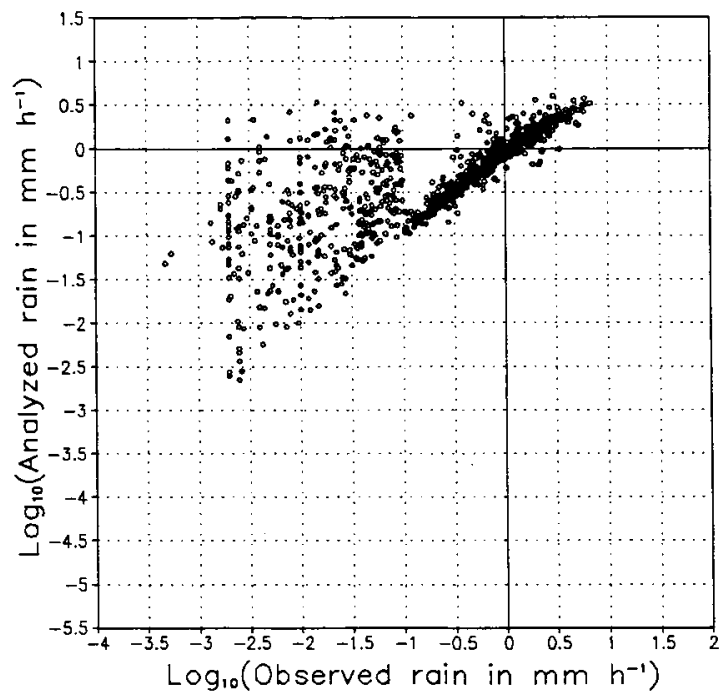

Figure 2. (a) Background rain versus observations at 1200 UTC 20 August 1998 . Shown are rain rates at1072 grid locations accepted by $\mathrm{O}-\mathrm{B} \mathrm{QC}$ checks for minimization. Not included are locations with no rain in either the background or the observation. (b) Analyzed rain rates given by (1) accepted by after-analysis QC checks versus observations. The scatter at very low rain rates reflects the large error standard deviations assigned to observed rain rates below $0.1 \mathrm{~mm} \mathrm{~h}^{-1}$ (See Sec. 2). 

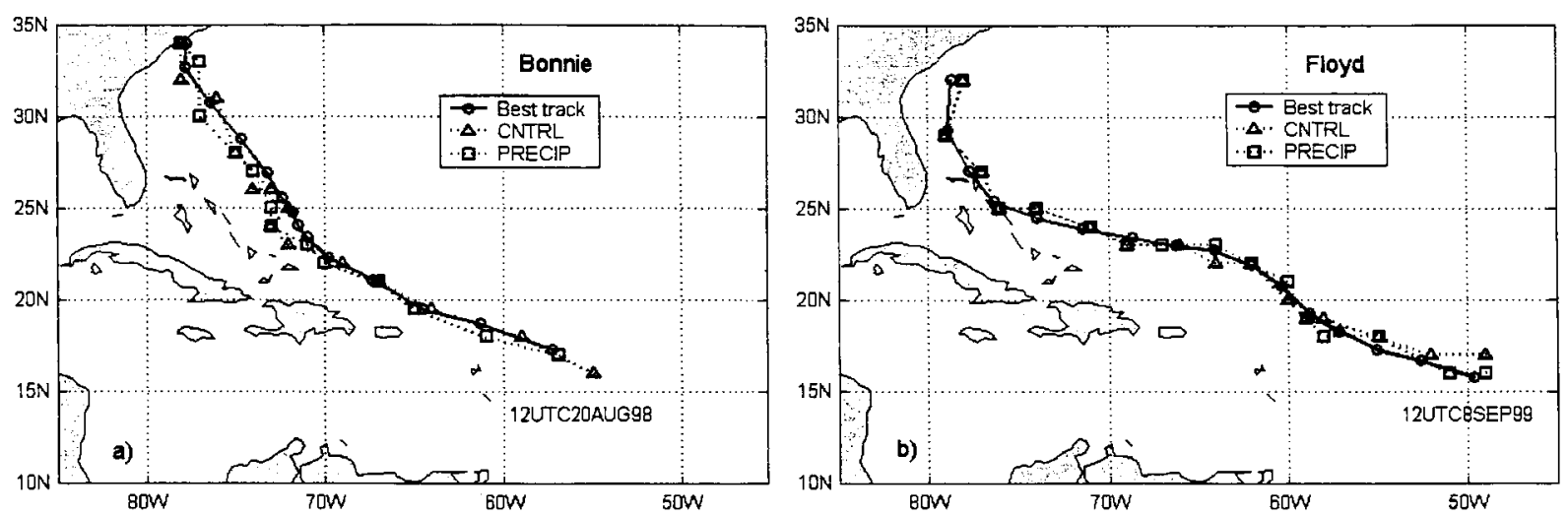

Figure 3. Analyzed 12-h positions of the minimum surface pressure of Bonnie and Floyd (rendered to the closest integer degree in latitude and longitude) from CNTRL and PRECIP analyses, compared with the NOAA best track locations. The first analysis time of the track is identified for each storm. 

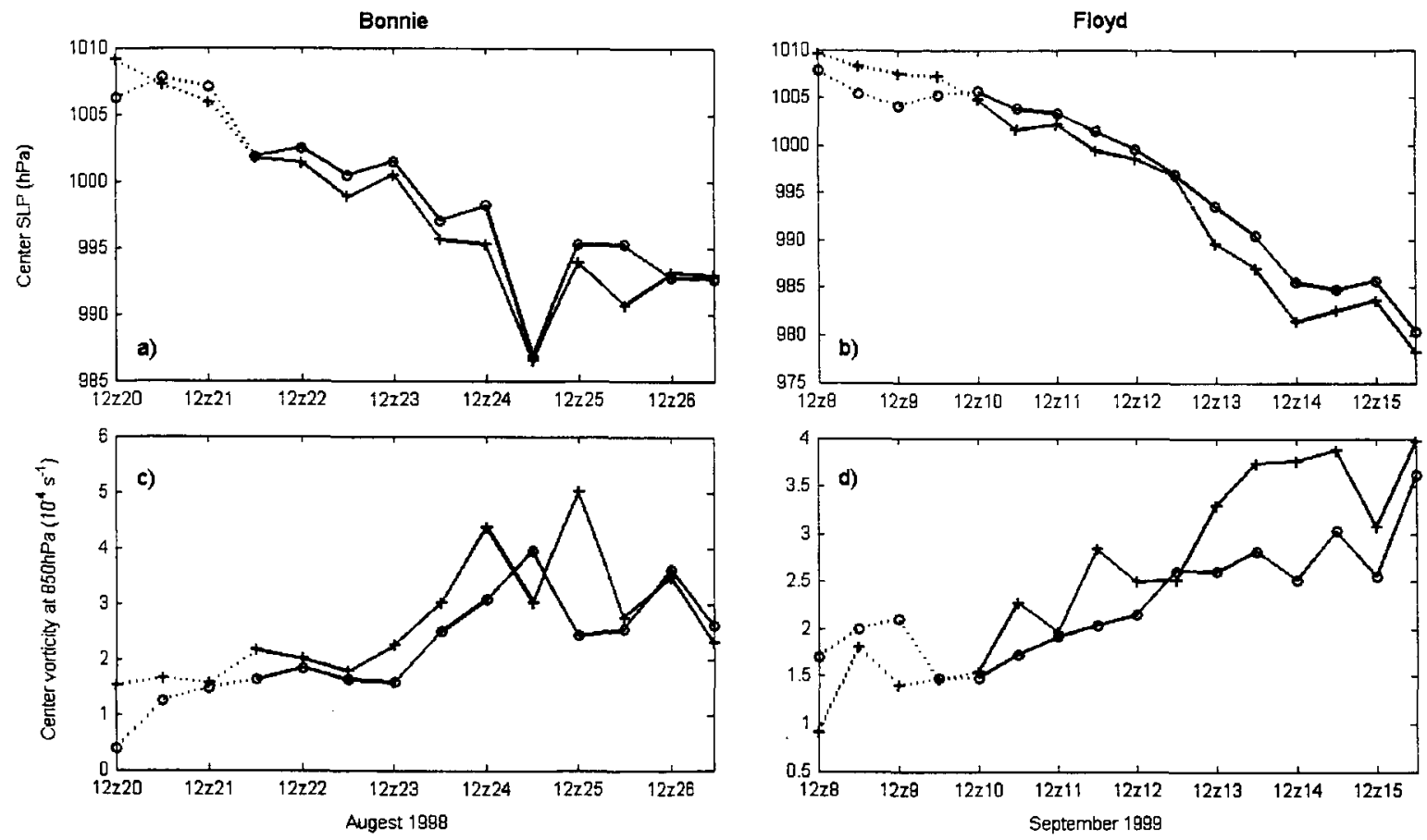

Figure 4. (a) Minimum sea-level pressure (SLP) along the track of Tropical Storm Bonnie (dotted lines) and Hurricane Bonnie (solid lines). The open circles show the CNTRL analysis and the crosses are the PRECIP analysis. (b) Same as (a) but for Floyd. (c) $850 \mathrm{hPa}$ vorticity along the Bonnie track. (d) Same as (c) except for Floyd. 

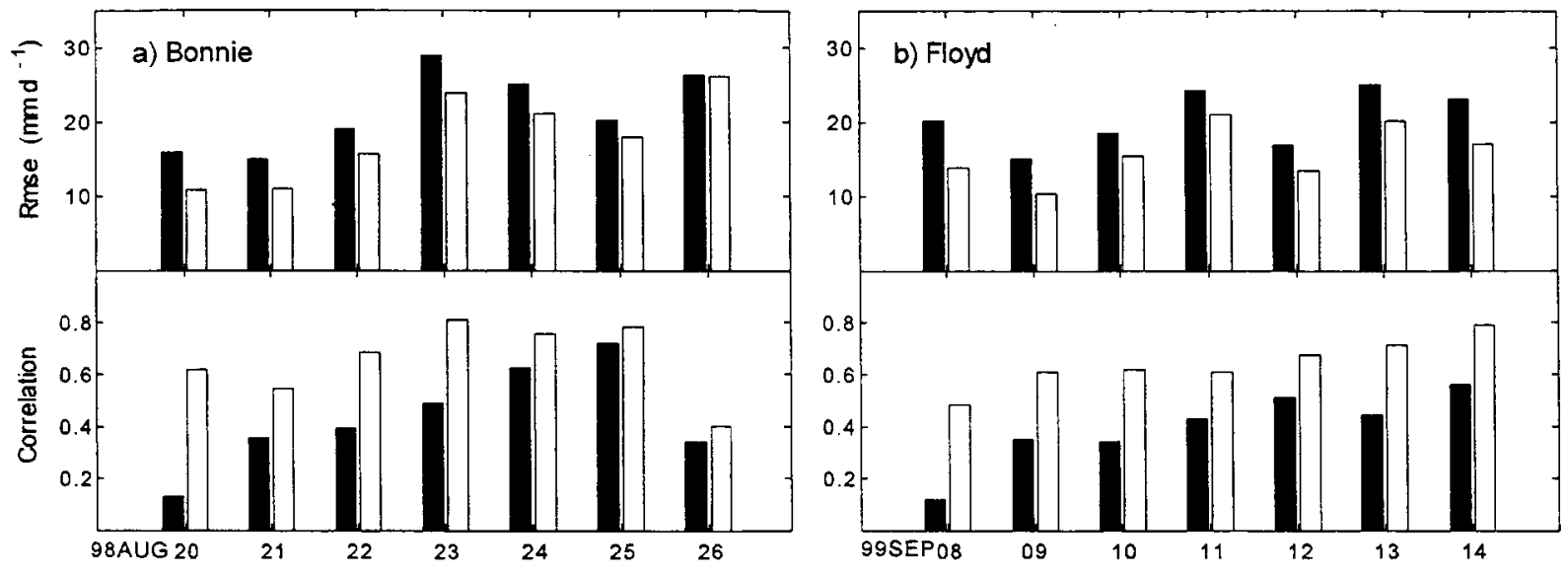

Figure 5. (a) Root-mean-square (rms) errors and spatial correlation of daily averaged rain rate over a $20^{\circ}$ latitude and $30^{\circ}$ longitude moving domain along Bonnie's track. The black and white bars refer to CNTRL and PRECIP analyses, respectively. (b) Same as (a) except for Floyd. 

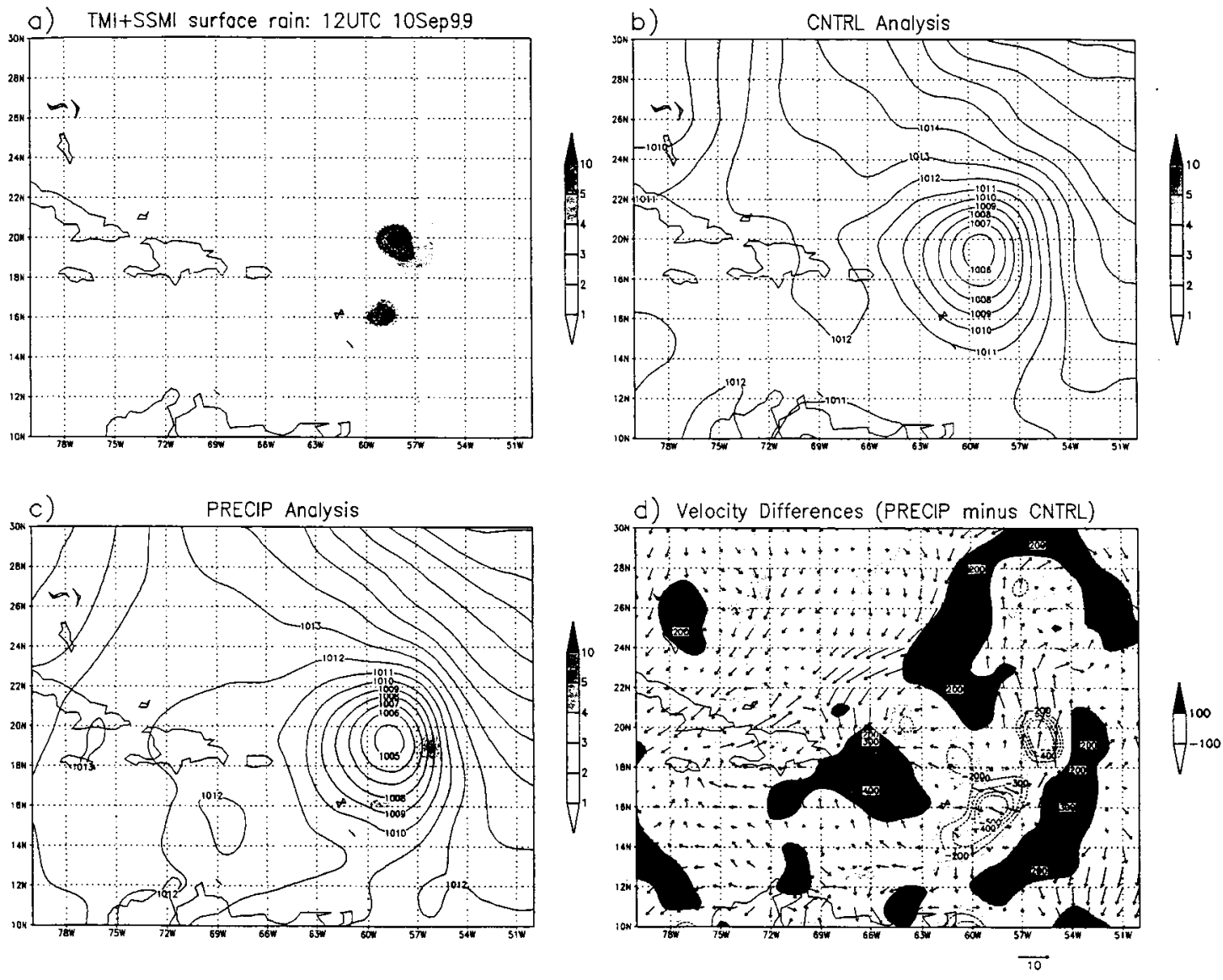

Figure 6. (a) Combined TMI and SSM/I observations of surface rain in $\mathrm{mm} \mathrm{h}^{-1}$ at 1200 UTC 10 September 1999. (b) Surface rain in $\mathrm{mm} \mathrm{h}^{-1}$ (shaded) and sea-level pressure in $\mathrm{hPa}$ (contours) in CNTRL at the same analysis time. (c) Same as (b) except for PRECIP analysis. (d) Changes in $500 \mathrm{hPa}$ omega velocity in $\mathrm{hPa}$ $\mathrm{d}^{-1}$ (shaded, with negative values indicating rising motion) and horizontal winds in $\mathrm{m} \mathrm{s}^{-1}$ at $200 \mathrm{hPa}$ between PRECIP and CNTRL analyses. The vector scale for $10 \mathrm{~m} \mathrm{~s}^{-1}$ is given for reference. 

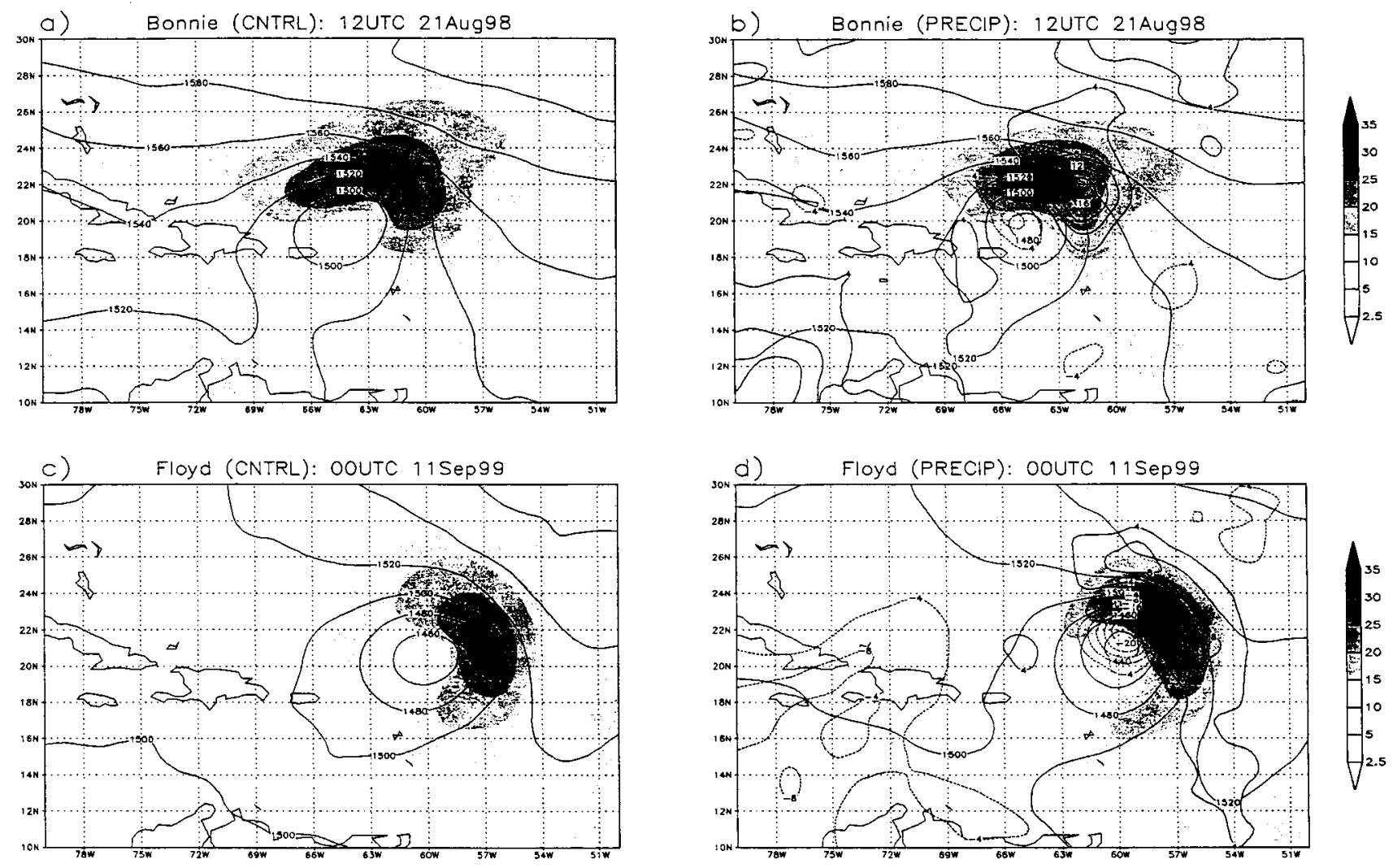

Figure 7. Analyses of $850 \mathrm{hPa}$ geopotential height in $\mathrm{m}$ (thick line) and wind speed in $\mathrm{m} \mathrm{s}^{-1}$ (shaded). (a) CNTRL analysis at 1200 UTC 21 August 1998 for Bonnie. (b) Same as (a) but for PRECIP analysis. The thin contours show PRECIP minus CNTRL differences in $850 \mathrm{hPa}$ geopotential height. (c) CNTRL analysis for Floyd at 0000 UTC 11 September 1999. (d) Same as (c) but for PRECIP analysis with the thin lines showing $850 \mathrm{hPa}$ geopotential height differences between PRECIP and CNTRL. 

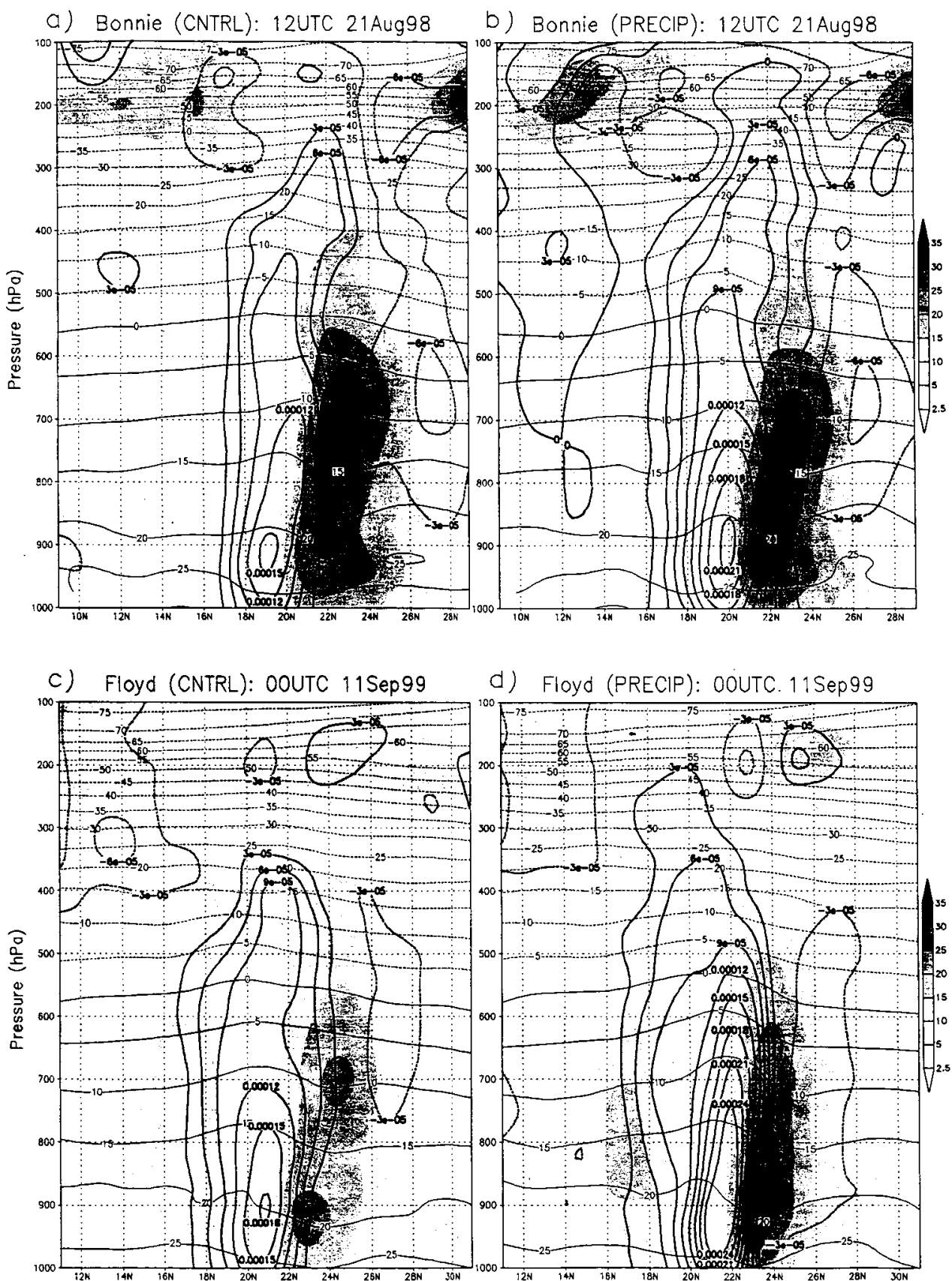

d) Floyd (PRECIP): OOUTC. 11 Sep99

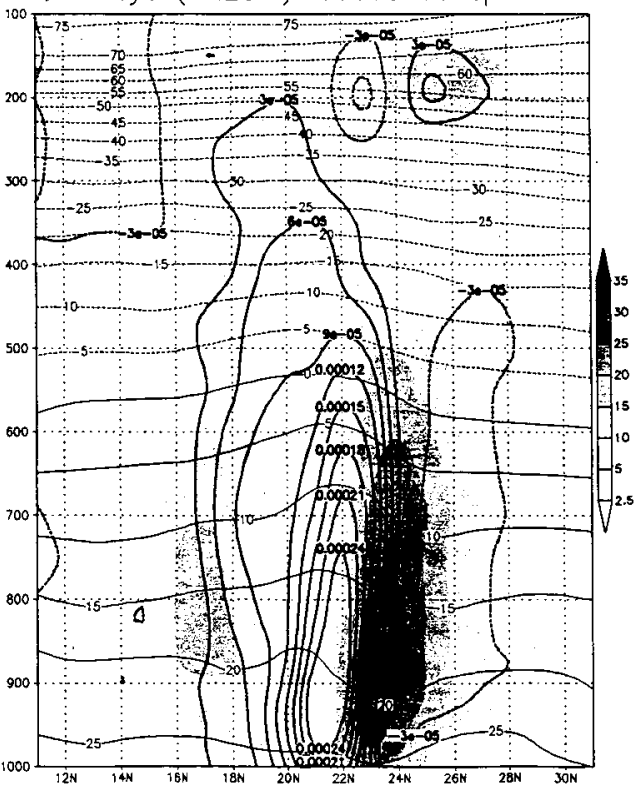

Figure 8. Upper panels: Meridional-vertical cross-sections at $65^{\circ} \mathrm{W}$ through the center of Bonnie at 1200 UTC 21 August 1998. Shown are wind speed in $\mathrm{m} \mathrm{s}^{-1}$ (shaded), relative vorticity in $\mathrm{s}^{-1}$ (thick lines at intervals of 3 $\mathrm{x} 10^{-5}$, with the zero contour omitted), and temperature in ${ }^{\circ} \mathrm{C}$ (thin lines). (a) CNTRL analysis. (b) PRECIP analysis. Lower panels: Similar cross-sections for Floyd at $60^{\circ} \mathrm{W}$ and 0000 UTC 11 September 1999. (c) CNTRL analysis. (d) PRECIP analysis. 

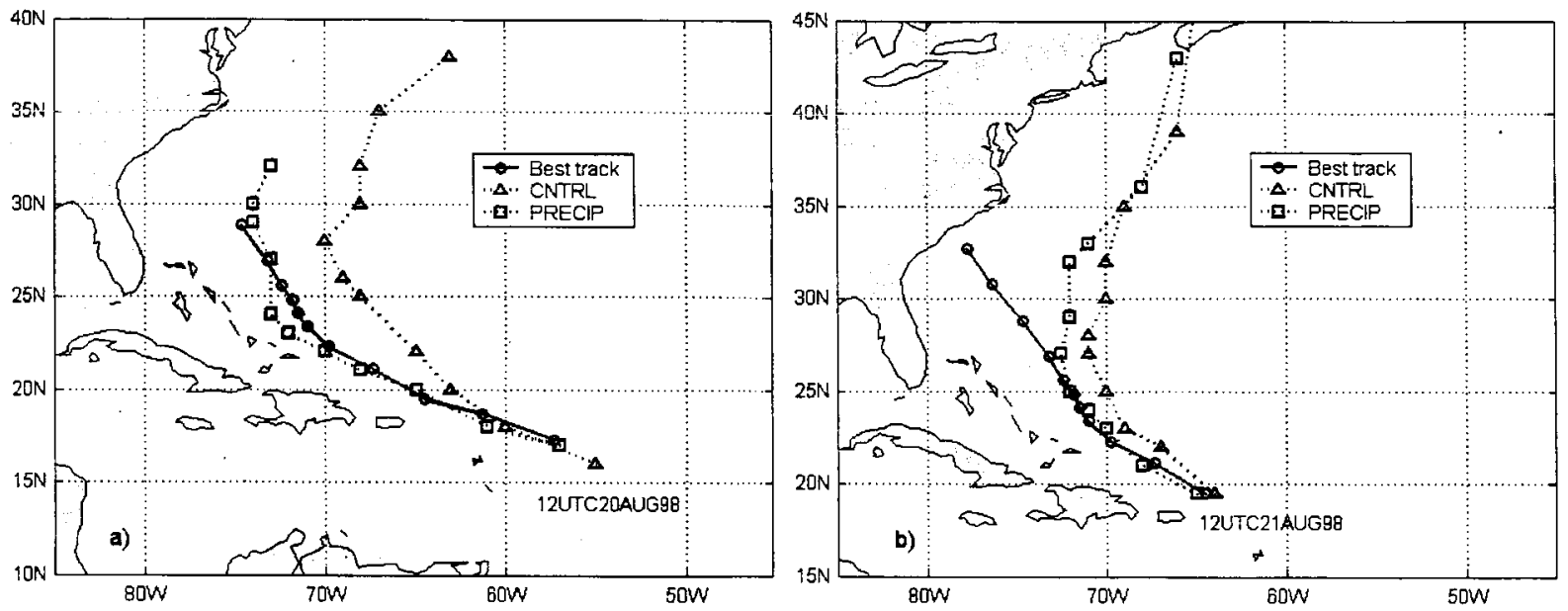

Figure 9. Five-day Bonnie track forecasts. Triangles and squares mark forecasts (dashes) initialized with CNTRL and PRECIP analyses, respectively. The storm positions are rendered to the closest integer degree in latitude and longitude. The circles show positions of the best track (solid). (a) Forecasts issued from 1200 UTC 20 August 1998. (b) Forecasts issued from 1200 UTC 21 August 1998. 

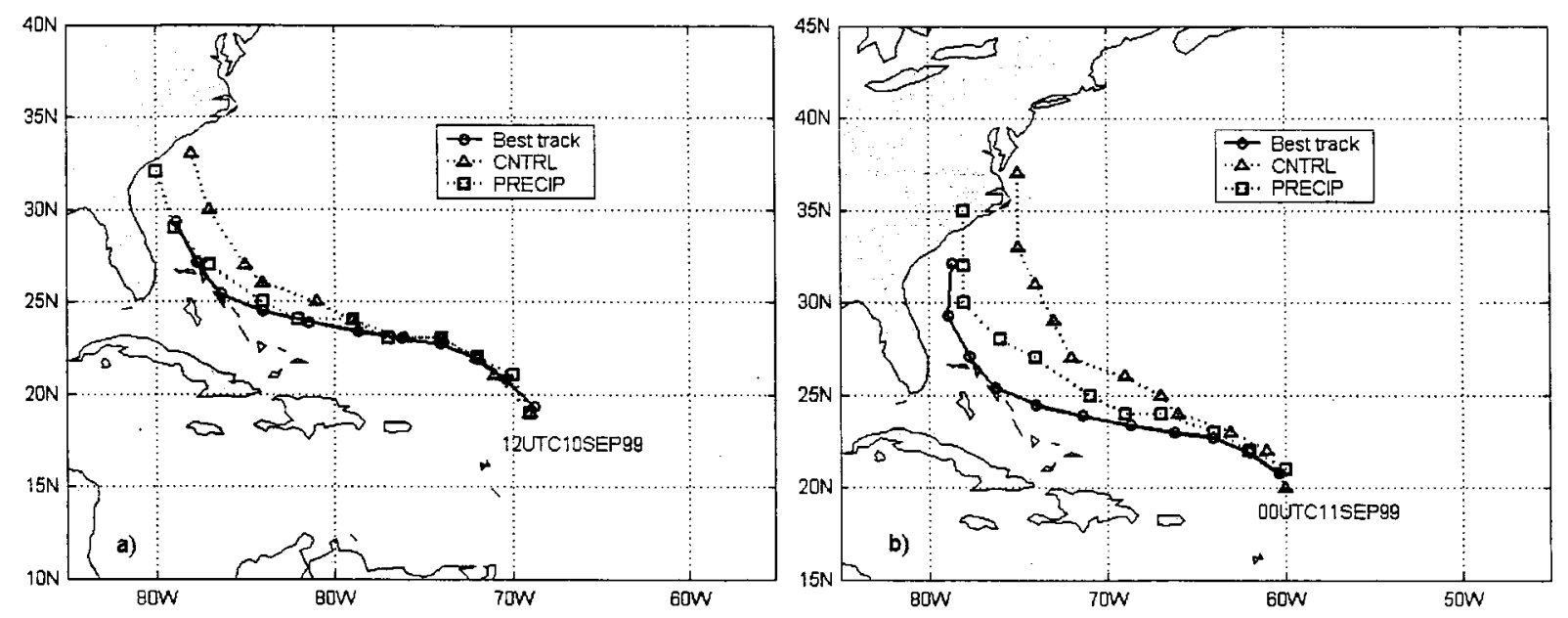

Figure 10. Five-day Floyd track forecasts. Triangles and squares mark forecasts (dashes) initialized with CNTRL and PRECIP analyses, respectively. The storm positions are rendered to the closest integer degree in latitude and longitude. The circles show positions of the best track (solid). (a) Forecasts initialized from 1200 UTC 10 September 1999. (b) Forecasts issued from 0000 UTC 11September 1999. 

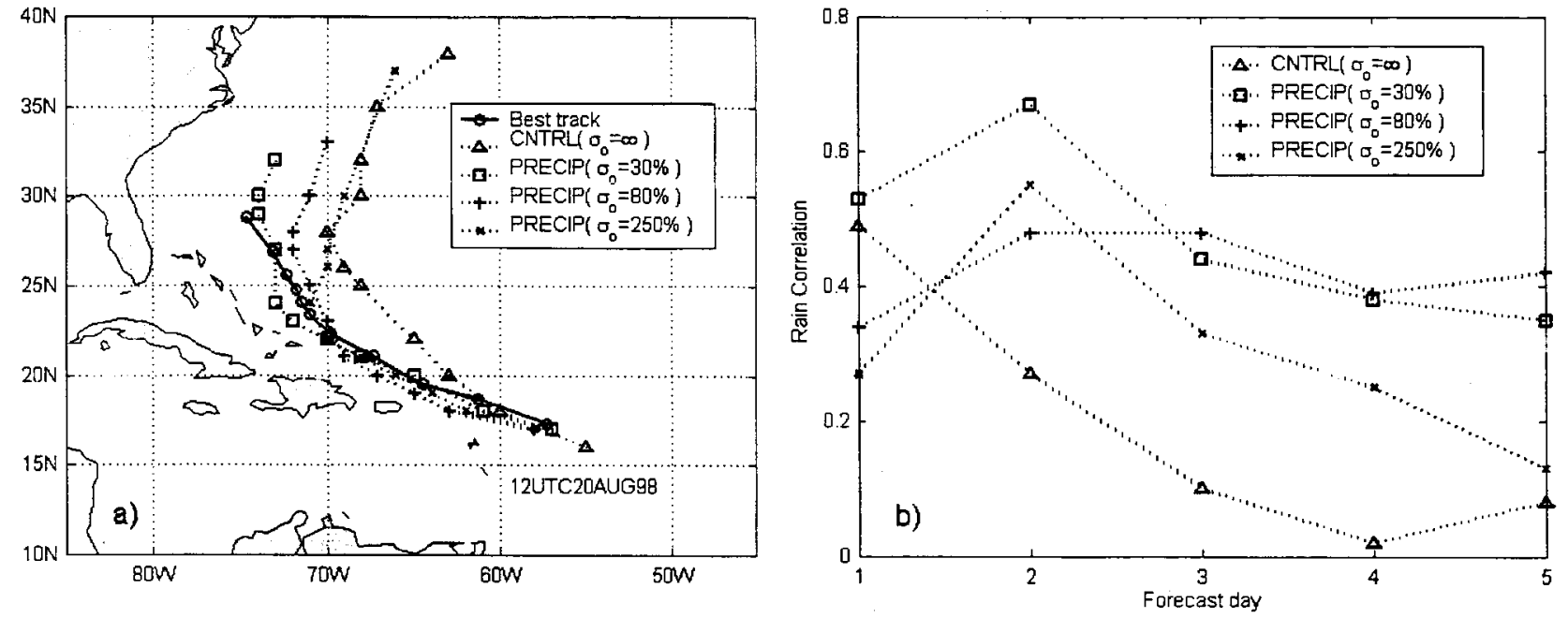

Figure 11. Sensitivity of a 5-day Bonnie forecast from 1200 UTC 20 August 1998 to TMI and SSM/I rainfall information retained in the initial condition. (a) Comparison of 5-day track forecast with the NOAA best track analysis. (b) Spatial correlations between 5-day precipitation forecasts and combined TMI and SSM/I daily rain rates. The legend identifies the forecasts by the error standard deviation for rain $\left(\sigma_{o}\right)$ in the analysis used for initial condition. 

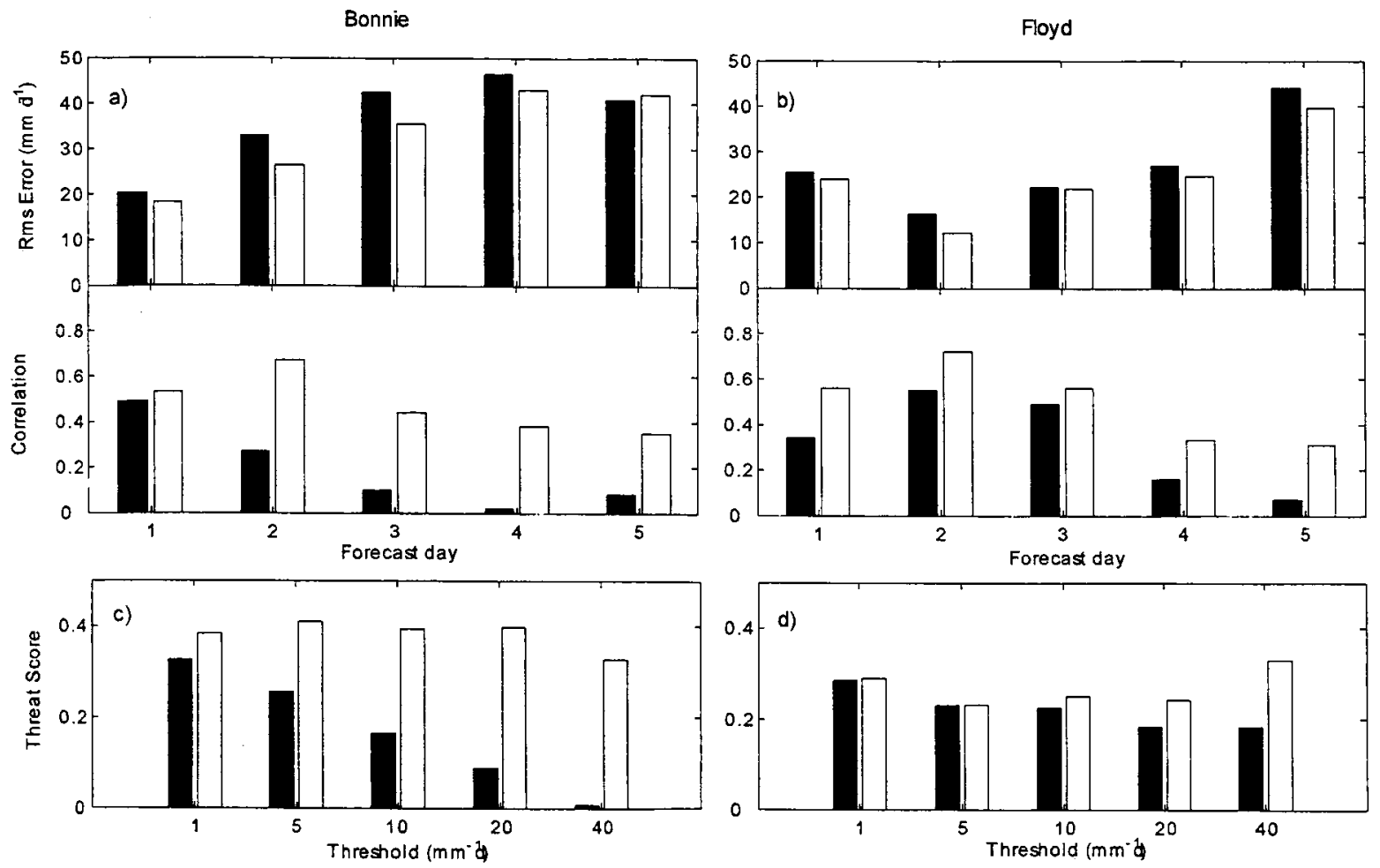

Figure 12. Root-mean-squar (rms) errors and spatial correlations of 5-day precipitation forecasts initialized with CNTRL analysis (black) and PRECIP analysis (white). (a) Bonnie forecast issued from 1200 UTC 20 August 1998. (b) Floyd forecast issued from 0000 UTC 11 September 1999. The statistics are for a $20^{\circ}$ latitude by $30^{\circ}$ longitude moving domain along the analyzed storm track. QPF Threat Scores for the Day 3 precipitation forecast are shown in (c) and (d) for Bonnie and Floyd, respectively. A higher Threat Score indicates greater forecast skills. 